\title{
The Value of the Oral Medicine Specialty in the Modern Healthcare Systems
}

\author{
Mohammed Bindakhil $^{\text {a }}$ Scarlet Charmelo-Silva ${ }^{a}$ Alhanouf A. Bin Dakhil ${ }^{b}$ \\ Ibrahim A. ALOmair ${ }^{\mathrm{C}}$ \\ aDepartment of Oral Biology and Diagnostic Sciences, Division of Oral Medicine, Augusta University, Augusta, \\ GA, USA; ${ }^{b}$ College of Medicine, Imam Mohammad Ibn Saud Islamic University (IMSIU), Riyadh, Saudi Arabia; \\ 'Department of Dermatology, College of Medicine, Imam Mohammad Ibn Saud Islamic University (IMSIU), Riyadh, \\ Saudi Arabia
}

\section{Keywords}

Multidisciplinary approach $\cdot$ Healthcare $\cdot$ Oral health · Oral medicine $\cdot$ Interprofessional integration

\begin{abstract}
Background: Oral medicine is a subdiscipline of dentistry that concerns itself with the oral health of patients; it focuses on providing diagnosis and management of oral and maxillofacial diseases, as well as direct dental care for those in medically complex situations. As primary healthcare providers, physicians and dentists are often the first to evaluate patients with orofacial symptoms and make diagnoses related to oral health, whether explicit or manifested through systemic diseases. However, a lack of familiarity with oral medicine among healthcare providers often leads to significant delays in the accurate diagnosis and appropriate treatment of many oral and maxillofacial disorders. It has been well established that a lack of training and education in oral health among physicians is an impediment to alleviating the situation. Summary: Several studies and scientific reports in the medical community have shown a greater professional interest in oral health and medicine, with numerous recent articles reflecting a clear rise in multiple factors: an increased interest in additional training and a rise in oral diseases. This review focuses on the need for a multidisciplinary approach
\end{abstract}

in modern healthcare systems that implements the oral medicine specialty. It also serves to emphasize the role of oral medicine in medical training and education, while simultaneously discussing the prevalence of oral diseases and awareness of oral health among physicians in Saudi Arabia. Key Messages: The prevalence of oral health conditions in Saudi Arabia appears to be high yet is accompanied by a scarcity of oral medicine specialists in this part of the world. Furthermore, numerous studies have revealed that physicians from different specialties in Saudi Arabia lack training in oral health and possess an insufficient awareness of the oral medicine specialty. This paucity of oral medicine specialists in Saudi Arabia presents a key issue as the clinical services and medical education provided by such specialists in healthcare systems can have a profound positive impact on each patient's quality of life and health outcomes, as well as the standards of physicians' training.

(c) 2021 The Author(s)

Published by S. Karger AG, Basel

\section{Introduction}

The American Academy of Oral Medicine (AAOM) defines oral medicine as a subdiscipline of dentistry that is primarily concerned with oral health, as well as the diagnosis and treatment of disorders or diseases affecting
C 2021 The Author(s)

Published by S. Karger AG, Basel

This is an Open Access article licensed under the Creative Commons Attribution-NonCommercial-4.0 International License (CC BY-NC) (http://www.karger.com/Services/OpenAccessLicense), applicable to the online version of the article only. Usage and distribution for commercial purposes requires written permission.
Correspondence to:

Mohammed Bindakhil, mbindakhil@augusta.edu 
the oral maxillofacial region [1]. Oral medicine encompasses the management of ulcerative and vesiculobullous diseases of the oral cavity, orofacial pain disorders, salivary gland diseases, regional neurological impairments, temporomandibular disorders, oral complications from systemic diseases, and oral potentially malignant disorders [2]. Over the last several years, oral healthcare has taken great strides and seen dramatic advancements [3].

Concerns regarding oral and maxillofacial health conditions - including oral mucosal diseases and pain in the orofacial region - are not limited to oral health specialists. These conditions are also relevant to other physicians as patients with such diseases often consult their physicians for advice and treatment options [4]. In particular, primary care, otolaryngology, and dermatology providers consult with such patients on a frequent basis. Despite the relative frequency of this occurrence, physicians are still found to have a lack of training with respect to oral and maxillofacial diseases and are unaware of the appropriate care for patients affected with these conditions [4]. To address this, there is a growing sentiment in both the medical and dental communities that greater integration between the 2 disciplines would be of great benefit to each healthcare system [4].

In order to successfully improve each patient's healthcare outcomes and quality of life, healthcare systems require well-trained specialists who work interprofessionally to provide comprehensive and continuous care [3]. In this review, we discuss the oral medicine specialty's role in healthcare systems, medical and dental education, and interdisciplinary patient care. Additionally, we discuss some of the challenges that patients and healthcare providers currently face regarding the appropriate care of oral health conditions. We also shed light on the prevalence of oral conditions and the awareness of oral health among physicians in Saudi Arabia. Furthermore, we discuss the current status of the specialty of oral medicine in this country, as well as strategies for a better integration between medicine and oral health in the Kingdom's healthcare systems.

\section{The Need for Oral Medicine in Saudi Arabia}

Alongside their dentists colleagues, physicians frequently encounter and make the initial diagnosis regarding the oral manifestations of systemic diseases, including diabetes, malignancies, and human immunodeficiency virus (HIV), among others [5]. Unfortunately, a physician's lack of familiarity with oral and maxillofacial dis- eases may lead to delayed or inappropriate diagnosis and/ or management of such illnesses [5]. Currently, there are no data available regarding the amount of time patients with oral conditions wait before they are evaluated by an oral medicine specialist in Saudi Arabia. Nevertheless, several retrospective studies have been carried out in the USA to assess the referral patterns and accessibility of oral medicine services. In order to have their oral conditions properly assessed and diagnosed by an oral medicine specialist, patients were found to have waited an average of 16.8 months and traveled twice as far for their clinical evaluation relative to their first referral appointment [57]. Many of such patients had also been evaluated by 1 to 4 or more additional clinicians before the oral medicine specialist referral [6].

Compared with North America and other developed parts of the world, Saudi Arabia has fewer oral medicine specialists [8]. There is currently no available database to indicate the actual numbers of local oral medicine specialists in Saudi Arabia. Nevertheless, a specialist search in this region using AAOM's online portal resulted in only 8 specialists in total. Almazrooa et al. [8] estimate that there are fewer than 100 oral medicine specialists in the Kingdom. However, due to a lack of official statistics, there is no reliable way to measure the rate of oral medicine specialists against Saudi Arabia's population.

The number of officially recorded dermatologists is similarly limited; this is significant as these are specialists frequently sought by patients suffering from oral disease. In the private sector and government hospitals combined, there are scarcely over 2,000 dermatologists, which results in a rate of 1 dermatologist per 15,000 people across all of Saudi Arabia [9]. It can safely be assumed that there are even fewer oral medicine specialists; therefore, the per capita rate will be much lower. Expanding postgraduate programs in oral medicine across Saudi Arabia should be a priority in addressing what is obviously a critical need.

Although the prevalence of oral diseases in Saudi Arabia is still understudied, some studies have attempted to address this topic. A recent study by Yenugadhati et al. [10] showed that $86.1 \%$ of cancer patients at a single center in Saudi Arabia experienced oral health conditions, including ulcerations, gingival bleeding, xerostomia, and bruxism. A similar study by Murshid et al. [11] found the oral mucositis prevalence among Saudi Arabian children with leukemia to be $23.3 \%$. Furthermore, Attas et al. [12] found 68\% of HIV-positive patients in Saudi Arabia to have oral lesions, compared to $4 \%$ in a healthy control group. Another study conducted by Al Wayli et al. [13] revealed that 5,215 female patients reported oral conditions over a 5-year
Bindakhil/Charmelo-Silva/Bin Dakhil/ ALOmair 
period at a single healthcare center. Among these conditions, the most common (in order) were pyogenic granuloma, infectious lesions, and oral lichen planus.

With only a handful of studies on oral cancer in Saudi Arabia to draw from, Basha et al. [14] performed a systematic review and found that in some places - particularly in areas where chewing tobacco was popular - the prevalence of oral cancer was as high as $21.6 \%$. Even worse, most cases were not diagnosed until late stages (stages III and IV), with staggeringly low 5 -year survival rates ranging from 12.9 to $24 \%$. This is lower than the rates found in the literature by at least $50 \%$, with 5-year survival being as high as $94 \%$ in cases that were diagnosed at an early stage [15]. Therefore, the early detection of oral cancer is of immediate concern in Saudi Arabia as this could directly improve survival rates for patients. However, such early detection is only possible through improving knowledge among healthcare providers regarding potentially malignant oral conditions. This discrepancy is highlighted in a study by Jaber et al. [16], which was carried out across multiple centers in Saudi Arabia to assess healthcare practitioners' perception of their oral cancer prevention and early detection training. The results showed that $45.1 \%$ saw their knowledge and training as being low, with $53.6 \%$ considering their knowledge to not be current. Fortunately, $76 \%$ of respondents correctly identified early detection as improving 5-year survival for oral cancers. Therefore, it is assumed that physicians in Saudi Arabia, despite not being well trained in the early detection of oral cancer, are aware of its importance and would be interested in expanding their clinical expertise in this field.

\section{Awareness of Oral Medicine among Healthcare Providers}

Despite its status as an emerging specialty, oral medicine is still better known and more integrated with general healthcare in the USA than it is in Saudi Arabia [8]. Generally, it is not just dental practices that refer patients to oral medicine specialists in the USA - typically, the fields of dermatology, internal medicine, and otorhinolaryngology, among others, are also involved in these referrals $[3,6,7]$. There appears to be a distinct lack of awareness or understanding of the oral medicine profession among medical service providers in the Middle East. Alrashdan et al. [17] carried out a study to this effect in Jordan. Of the 1,492 physicians interviewed, only 52\% were even aware that oral medicine was an independent specialty. A similar result was found by Almazrooa et al.
[8], who assessed Saudi Arabian medical practitioners regarding their knowledge and the availability of oral medicine, receiving 65 physician responses. Only $60 \%$ were aware of oral medicine specialists, and a further half of those had never referred patients to an oral medicine specialist [8]. Nevertheless, Almazrooa el al. [8] reported that $83.3 \%$ of physicians who referred patients to oral medicine in Saudi Arabia had a satisfactory experience. Within this study, Almazrooa et al. [8] found the following to be most challenging for Saudi Arabian physicians to diagnose and/or manage: nonspecific oral ulcers, oral lichen planus/lichenoid reactions, graft-versus-host disease, potentially malignant oral lesions, and vesiculobullous diseases. The findings from Alrashdan et al. [17] agree with this sentiment, indicating that oral ulcers have the highest rate of physician referral to oral medicine (72\%) followed by white or red lesions (66\%).

Awareness of oral medicine varied among the types of medical specialists. Both Alrashdan et al. [17] and Almazrooa et al. [8] showed that dermatologists were the providers who were the most likely to understand oral medicine and what the field entails. Almazrooa et al. [8] also demonstrated that $50 \%$ of oral medicine referrals were made by dermatologists likely because these specialists frequently encounter oral mucosal conditions. Somewhat surprisingly, primary care physicians demonstrated the least awareness of oral medicine as a specialty. Alrashdan et al. [17] reported that a mere $37 \%$ of family medicine specialists possessed an awareness of oral medicine, while Almazrooa et al. [8] found that only $16 \%$ of oral medicine referrals were made by a primary care physician.

\section{Oral Health Components in Medical Education}

It is evident that physicians themselves have the potential to greatly improve the quality of and access to oral healthcare for patients; however, this is dependent on such physicians receiving the proper training [4]. Currently, allopathic family medicine and emergency residency programs in the USA are required to include practical oral healthcare education and hands-on experience in their curricula. Additionally, the American Board of Family Medicine certification examination now includes oral health-related questions [4]. In contrast, the Saudi Board for Family Medicine curriculum for 2020 shows no indication of oral health components except for "tongue-tie; cleft lip and palate in children" under otolaryngology topics [18]. In a recent study, Alshathri et al. [19] aimed to discern general oral health knowledge among family physicians in Saudi Arabia. 
Their findings show that only $7.19 \%$ of family physicians in Saudi Arabia received any oral health training, revealing that this form of training is not provided to the vast majority of Saudi primary care practitioners. This lack of training in oral health may explain why family physicians in Saudi Arabia were among the least likely to be aware of oral medicine or appropriately refer patients with oral diseases in Almazrooa et al.'s study [8].

Nevertheless, evolving evidence points toward a growing professional interest in the inclusion of oral health components in medical training curricula. For instance, in the Journal of the American Board of Family Medicine, several recent articles demonstrate a renewed interest among medical practitioners in additional oral health training, as well as emphasizing the increase in dental and oral disease in their patient population [20-23]. Furthermore, the latest issue of the well-recognized Dermatologic Clinics series was entitled "Oral Medicine in Dermatology" and focused on providing knowledge concerning oral mucosal diseases to practicing dermatologists [24].

In a 2008 joint expert panel report, the American Association of Medical Colleges published a set of oral health learning objectives to prepare both dental and medical graduates to receive training in clinical settings [25]. This report also aimed to bring more attention to the intersectoral competence of all health practitioners undergoing training in order to encourage shared perceptions, expertise, and skills required for successful practice and interdisciplinary collaboration in the modern healthcare environment [25]. The Institute of Medicine's (IoM) report, Advancing Oral Health in America, has also given these oral health issues heightened priority [26]. The report made clear the critical role that education and clinical training play in improving physicians' health literacy, leading to the improvement of patient care outcomes [26].

In 2011, an expert panel of educators in the dental and medical communities embarked on the same mission [27]. They released an education report that listed the requisite knowledge, abilities, and attitudes that would be applicable for medical and dental students regarding the identification, management, appropriate referral of oral health conditions, and overarching importance of this multidisciplinary approach. In the report, the recommendations emphasized that the oral health of patients was a shared responsibility between both medical and dental professionals, which reflects the panel's belief in the importance of professional integration between the 2 disciplines [4, 27]. Despite a slew of calls for improved integrative healthcare that encompasses oral health from scientific organizational reports, published research, and a heightened sense of need among healthcare providers, progress continues to be slow. This sluggish improvement is due to numerous complicating factors, ranging from financial needs to inadequate workforce to inefficient bureaucracy, among many others.

\section{Oral Medicine in Healthcare Systems and Medical Centers}

The impact that oral medicine specialists have on patient care outcomes is well documented, and the profoundly positive results highlight the necessity of the field [28]. An interesting study was recently conducted by Sun et al. [5] at the University of Pennsylvania, which evaluated the services provided by the oral medicine practice. This study reported that of 6,024 outpatient oral medicine visits, the most commonly diagnosed conditions were those related to orofacial pain (46.63\%), followed by benign oral mucosal diseases (35.51\%) [5]. Additionally, the authors stated that the total number of patients who received services at oral medicine clinics is indeed higher than these statistics indicate as the study only reviewed cases involving residents and did not include cases that were only seen by attending oral medicine providers.

The importance of integrating oral medicine specialists into healthcare systems is exacerbated by the need to provide adequate in-patient care. Oral medicine specialists are frequently consulted by physicians for the evaluation and management of orofacial pain and oral mucosal diseases, including therapy-induced mucositis, graft-versus-host disease, vesiculobullous diseases, and soft tissue lesions. Furthermore, they are consulted in order to gain dental clearance before cardiac surgeries, chemotherapy, and head and neck radiation. Sun et al. [5] reported that oral medicine specialists were most commonly consulted for patients admitted in cardiovascular service (38.99\%), followed by oncology/hematology service (19.05\%).

Furthermore, dental treatment for medically complex patients is another essential role that oral medicine specialists can assume in medical centers. Such care should be provided on a case-by-case and personalized basis, as well as by a dental professional with an adequate background in medicine [29]. When providing treatment to medically complex patients, a dental professional should perform patient and risk assessments, as well as clinical evaluation. They should also develop treatment plans while taking into account the prioritization, goals, and complications of dental therapy [29]. The medical history of such patients may impact the delivery of dental treatment; additionally, factors such as wound healing, infection, bleeding, and drug
36

Saudi J Health Syst Res 2021;1:33-40 DOI: $10.1159 / 000515358$
Bindakhil/Charmelo-Silva/Bin Dakhil/ ALOmair 
interactions must be considered [29]. Furthermore, treatment modifications may be implemented during invasive dental procedures, such as prophylactics, preoperative, perioperative, and postoperative adjunctive measures [29].

The single-center study conducted by Sun et al. [5] showed that 1,648 medically complex patients who underwent dental treatment in the department of oral medicine had a median of 3 medical morbidities. Of these patients, the most frequent medical condition was cardiovascular disease, followed by immunodeficiency disorders, including HIV [5]. There is a lack of literature regarding the dental management of medically complex patients or hospital-based dentistry in Saudi Arabia, although such services are currently being provided in some of the country's large tertiary hospitals. Nevertheless, the recent study by Almazrooa et al. [8] showed that $73 \%$ of Saudi Arabian medical practitioners would request dental clearance for their patients before chemotherapy or radiotherapy; however, only $42 \%$ would do so prior to bisphosphonate therapy, and $52 \%$ were fully unsure. These figures indicate the stark, distinct lack of understanding regarding the maxillofacial complications of bisphosphonate therapy, such as osteonecrosis of the jaw, and the need of specialized dental care of such patients. The impact of oral health and its comorbidities on a patient's overall well-being must be comprehensively understood by healthcare providers.

Robust research is still needed in many areas of oral medicine, including potentially malignant disorders, dermatologic diseases of the oral cavity, and orofacial pain. Despite this, oral medicine specialists have made distinguished contributions to the current scientific research concerning oral health. A recent study by Stoopler et al. [30] assessed the scholarly productivity of graduates from a single oral medicine residency program in the USA. The authors found that $74 \%$ of the oral medicine graduates reported scholarly productivity - of those, $88 \%$ published chapter(s)/textbook(s) and $65 \%$ published research articles in scientific journals. Additionally, $48 \%$ reported receiving funding for clinical or basic science research, totaling to more than USD 5,000,000. Significant clinical research achievements in the field of oral medicine will be more feasible when the specialty is integrated within the healthcare systems, where interprofessional patient care occurs.

\section{Oral Medicine Residency Programs}

Many parts of the world are now home to accredited oral medicine training programs, featuring established goals for the field, core competencies, and experienced

Interdisciplinary Approach to Healthcare practitioners [3]. The global establishment and widespread recognition of the field will further increase awareness of and access to oral medicine for patients and practitioners alike. In the USA, the field has been emerging as a crucial subdiscipline of dental education, research, clinical care, and primary care for more than 50 years [31]. However, it was only in March of 2020 that major strides began to take place when the American Dental Association (ADA) approved the specialty recognition application of the AAOM, allowing it to adopt oral medicine as a dental specialty [32]. Only a few years prior to this, the Saudi Board of Oral Medicine and Oral Pathology was established in 2016 and recognized in that year by the Saudi Commission for Health Specialties [18].

Saudi Arabia's first oral medicine and pathology program was developed with the goal of producing wellqualified clinicians for patient management and hospital duties [18]. The program's description describes it as a 4-year program, combining didactic clinical science and training [18]. The program is residency-based in main tertiary hospitals throughout Saudi Arabia, combining specialized training in general medicine with training in oral medicine and pathology [18]. This includes dental training that extends to hospital dentistry and medically complex dental patient management, such as patients with cancer, organ transplants, and hematological disorders [18]. Saudi Arabian oral medicine residency graduates receive clinical training in several medical specialties, just as they would in the USA. These include hematology/ oncology, otolaryngology/head and neck oncology, infectious diseases, rheumatology, dermatology, and pain management [18]. In rotations for each subdiscipline, residents will partake in physical evaluation, diagnosis, and patient management.

In the UK, pursuing a medical and dental degree together shortens oral medicine training from 5 to 3 years. Given that medical approaches to oral conditions are on the rise and that an extensive medical background is required in the field of oral medicine, it has been suggested that oral medicine be classified as a subdiscipline of medicine, rather than dentistry in the USA [33]. Such proposal is mainly based on that merely expanding medical focus in dental schools to account for oral medicine which faces the obstacle of lack of curriculum space and time constraints for the field to be properly learned, noting that the majority of oral medicine programs in the USA are already housed in dental schools [33]. Dental education could, of course, benefit from such additional focus in medicine, whether the oral medicine specialty changes to be a subdiscipline of medicine or not as training in 
medicine will be useful to dentists who encounter patients with health comorbidities that can complicate dental treatments. Furthermore, several dental specialties dental graduates could pursue involve/require extensive medical knowledge, including oral medicine, dental anesthesiology, and oral and maxillofacial surgery. While most training programs in such specialties lie under the umbrella of dental schools, they typically have an affiliation with medical centers in which residents spend a significant part - if not most - of their training, that is, oral medicine residencies in the USA. In Saudi Arabia, 4 centers are accredited to hold oral medicine and pathology residencies; of those, 3 are tertiary medical centers [18].

The presence of oral medicine services and training programs in medical centers can help compensate for the lack of medical experience in dental education. Through clinical rotations and externships in oral medicine, dental students will have the opportunity to spend time in a medical environment where oral health and medicine are integrated. Such exposure will further provide dental students with an overview of one of the career paths in oral medicine. In a study by Alrashdan et al. [34], it was found that senior dental students from a single Jordanian institution demonstrated a lack of interest in postgraduate oral medicine training. This study highlights a greater trend: there are very few established training programs in oral medicine and a lack of career guidance regarding oral medicine opportunities, thus affecting the desire of dental graduates to pursue this field [35]. Training in oral medicine allows graduates to practice in a range of environments, from a medical center to an educational facility to private practice [3]. In response to the higher demand for oral medicine specialists and the potential graduate interest, dental schools should emphasize oral medicine as a viable career path to upcoming graduates and provide them with information on these opportunities [35]. In Saudi Arabia, nevertheless, the number of new graduates who are interested in the field is experiencing a notable increase [18]. Therefore, there is a prominent need to address this growing interest by implementing a greater focus on local oral medicine training [18].

\section{Strategies to Integrate Oral Medicine into Healthcare Systems}

At this stage, the healthcare system as a whole would benefit greatly from the entrance of new oral medicine specialists who can provide high-quality care and better access to oral health. The best way to establish a consistent entry of oral medicine specialists into healthcare systems is to provide a recognizable presence among medical, health, and dental professional groups, as well as ensuring pathways for service reimbursement [3]. According to a study involving oral medicine clinicians from across the world published by Stoopler et al. [28], hospitals and dental schools are currently the most common settings in which oral medicine specialists are found. The authors also added that roughly half of the oral medicine specialists are participants in multidisciplinary oncology clinics/ services, while a majority are involved in diagnosing and managing different oral and maxillofacial diseases in patients [28].

According to the Ministry of Health's 2020 statistics, Saudi Arabia is home to 164 private hospitals and 334 public hospitals [9]. The Kingdom's healthcare has undergone considerable reform and modernization efforts, as evidenced by the Saudi Vision 2030. In 2019, the healthcare budget increased to 1.106 billion Saudi riyals (294.79 billion USD); since then, new healthcare insurance plans have been rolled out, and both free national telehealth and preventive care have seen notable upticks even before the ongoing pandemic of coronavirus disease 2019. In light of these important efforts for achieving state-of-theart healthcare in the Kingdom, the integration of oral medicine into the healthcare system would be of considerable positive impact.

In order to ensure the field's growth and patient access, it is essential that oral medicine services be funded under the medical model as a crucial part of integrated healthcare services [3,36]. Stoopler et al. [28], in the aforementioned international study, reported that the most common source of patient funding in oral medicine services was through government sources, followed by self-pay with no insurance. In contrast, reimbursement through medical or dental insurance was the least common source. Real change in the oral medicine funding model may currently be on the horizon due to broad support from medical organizations and a marked improvement in the public's understanding and view of oral health [36]. This interprofessional integration of the oral medicine specialty translates into improved healthcare efficiency and outcomes while simultaneously reducing overall healthcare costs.

The nonexistent data regarding the number of oral medicine specialists in Saudi Arabia demonstrate the critical need for such online tools in the region, through which patients and healthcare providers can locate nearby appropriate specialists for their needs [35]. Efficient communication through online platforms will facilitate
Bindakhil/Charmelo-Silva/Bin Dakhil/ ALOmair 
referrals to oral medicine specialists, thus allowing for further, more rapid adoption. Furthermore, a simple and easily accessible online platform that lists accredited training programs and career opportunities would greatly benefit dental students and recent graduates interested in the oral medicine field.

Moreover, oral medicine specialists in Saudi Arabia should begin expending great efforts to introduce their specialty to the medical field and assert the necessity of their services. Outreach to hospitals within the Kingdom and providing continuing education to Saudi societies and professionals in the relevant medical specialties can help achieve specialty awareness [8]. Additionally, introducing the oral medicine specialty to current medical curricula and residency programs would further establish progress, allowing for residents in medical specialties to rotate through oral medicine clinics.

Strategies to integrate oral health and medicine can include the promotion of interprofessional collaboration as implemented by numerous North American medical schools - to align dental schools with other professions, including medicine and nursing [17]. Other relevant strategies are as follows: the pairing of medical and dental students in ground rounds/seminars and dental rotations in oral medicine services for medical students, and ensuring medical and dental students have collaborative research activities at the early stages to promote interprofessional collaboration [17].

Furthermore, proper physician training in oral health can be achieved during medical residencies in a number of ways. These include rotations in oral medicine for medical residents in specialties of high relevance to oral medicine practice, such as dermatology, otolaryngology, internal medicine, and family medicine $[8,17]$. Such high-standard, interprofessional training of medical residents is only feasible through the presence of oral medicine practices/programs in the places where all medical training and patient care occur. Not only will this allow physicians to effectively include oral health practices in their preventive approaches but it would also endow them with the skills needed to provide appropriate counseling, treatment, referrals, and support to their patients through interdisciplinary expertise [4].

\section{Conclusion}

Oral medicine, as a specialty primarily concerned with oral health, is an essential part of healthcare education and the integration of oral health and medicine [2].
Oral medicine specialists typically collaborate on a regular basis with primary care physicians, dental professionals, and many other healthcare providers in order to provide care to patients with a complex array of morbidityrelated needs [5]. This role places oral medicine specialists in a unique and important position - they straddle the line between oral and systemic health, functioning in the realms of both clinical dentistry and medicine [5]. Practitioners of oral medicine must be highly knowledgeable and skilled in a number of cross-disciplinary fields including dermatology, infectious diseases, internal medicine, rheumatology, and pharmacology, among others [29]. Oral health-related training for physicians will allow healthcare providers to offer better emergency care, patient counseling, and treatment of maxillofacial and oral conditions, leading to the overall improvement of the public's oral health [4]. Recognition for the value of the oral medicine specialty in healthcare systems has already begun to take place. This has occurred in a number of contexts, thus validating its importance as a field and ensuring its place among medical centers and educational institutions around the world. Overall, this will undoubtedly improve the quality and access to healthcare services for the public while simultaneously deepening the interdisciplinary approach taken by healthcare providers.

\section{Conflict of Interest Statement}

Mohammed Bindakhil is employed as an assistant professor of Oral Medicine at Augusta University, Augusta, GA, USA. Scarlet Charmelo-Silva is employed as an assistant professor of Oral Medicine at Augusta University, Augusta, GA, USA. Alhanouf A Bin Dakhil reports no conflict of interest. Ibrahim A. ALOmair is an assistant professor and aonsultant in Dermatology and Laser and Dermatologic Surgery in the Department of Dermatology, College of Medicine, Imam Mohammad Ibn Saud Islamic University (IMSIU), Riyadh, Saudi Arabia.

\section{Funding Sources}

Funding was not received for this research.

\section{Author Contributions}

Mohammed Bindakhil: design, writing, editing, and literature review; Scarlet Charmelo-Silva: design, writing, and revision; Alhanouf A Bin Dakhil: design and writing; Ibrahim A. ALOmair: design, writing, and supervision. 


\section{References}

1 American Academy of Oral Medicine [cited 2020 October 21]. Available from: www. aaom.com.

2 Stoopler ETDe Rossi SS, Greenberg MS, Sollecito TP. The Global footprint of oral medicine specialists: the University of Pennsylvania experience. J Dent Educ. 2016;80(12): 1464-7.

3 Pinto A, Mendes RA. The business model of oral medicine-new market disruption of the practice of dental medicine. Oral Surg Oral Med Oral Pathol Oral Radiol. 2019;127(2): 89-91.

4 Cohen LA. Expanding the physician's role in addressing the oral health of adults. Am J Public Health. 2013;103(3):408-12.

5 Sun M, Sollecito TP, Greenberg MS, Pinto A, Stoopler ET. Analysis of clinical oral medicine practices at the University of Pennsylvania: a 5-year retrospective study. Oral Surg Oral Med Oral Pathol Oral Radiol. 2020;129(3): 215-e6.

6 Villa A, Stock S, Aboalela A, Lerman MA, Woo SB, Sonis ST, et al. Oral Medicine referrals at a hospital-based practice in the United States. Oral Surg Oral Med Oral Pathol Oral Radiol. 2015;119(4):423-9.

7 Pinto A, Khalaf M, Miller CS. The practice of oral medicine in the United States in the twenty-first century: an update. Oral Surg Oral Med Oral Pathol Oral Radiol. 2015; 119(4):408-15.

8 Almazrooa SA, Binmadi NO. The knowledge and attitude of physicians' toward the oral medicine specialty. J Oral Maxillofac Surg Med Pathol. 2021;33(1):83-8.

9 Ministry of Health - Kingdom of Saudi Arabia. Statistical yearbook $1440 \mathrm{H} / 2020$ [cited 2020 December 25]. Available from: https:// www.moh.gov.sa/en/Ministry/Statistics/ book/Pages/default.aspx.

10 Yenugadhati N, Albalawi AN, Qureshey AT, Qureshey ET, Al-Jahdali H, Jazieh AR, et al. Associated factors for oral health problems in a sample of Saudi cancer patients. Cancer Manag Res. 2018;10:1285-93.

11 Murshid EZ, Azizalrahman TA, AlJohar AJ. Oral mucositis in leukemic Saudi children following chemotherapy. Saudi J Dent Res. 2017; 8(1-2):79-85.

12 Al-Attas SA. Prevalence of oral and peri-oral lesions among a group of Saudi HIV-infected patients. Saudi Med J. 2014;35(9):1113-9.
13 Al Wayli H, Rashed BAWK, Kumar A, Rastogi $\mathrm{S}$. The prevalence of oral mucosal lesions among Saudi females visiting a tertiary dental health center in Riyadh region, Saudi Arabia. J Int Oral Health. 2016;8(6):675-8.

14 Basha S, Mohamed RN, Al-Thomali Y, Shamrani A. The prevalence of oral cancer in Saudi Arabia : a systematic review. Ann Med Health Sci Res. 2019;9.

15 Sciubba JJ, Larian B. Oral squamous cell carcinoma: early detection and improved 5-year survival in 102 patients. Gen Dent. 2018 NovDec;66(6):e11-e6.

16 Jaber L, Shaban S, Hariri D, Smith S. Perceptions of healthcare practitioners in Saudi Arabia regarding their training in oral cancer prevention, and early detection. Int J Health Care Qual Assur. 2011;24(1):8-18.

17 Alrashdan MS, Darwazeh AMG, Hassona Y, Bader DH, Khader YS. Awareness of oral medicine among medical practitioners, evidence of the unbridged interdisciplinary gap. J Eval Clin Pract. 2019;25(1):142-7.

18 The Saudi Commission for Health Specialties [cited 2020 Oct 22]. Available from: https:// www.scfhs.org.sa.

19 Alshathri B, Aljasser N, Kofi M. Knowledge of oral health among family medicine physicians in Riyadh, Saudi Arabia 2020. J Family Med Prim Care. 2020;9(9):4761-8.

20 Edwards PC, Kanjirath P. Recognition and management of common acute conditions of the oral cavity resulting from tooth decay, periodontal disease, and trauma: an update for the family physician. J Am Board Fam Med. 2010;23(3):285-94.

21 Davis MM, Hilton TJ, Benson S, Schott J, Howard A, McGinnis P, et al. Unmet dental needs in rural primary care: a clinic-, community-, and practice-based research network collaborative. J Am Board Fam Med. 2010; 23(4):514-22.

22 Mungia R, Funkhouser E, Makhija SK, Reyes SC, Cohen RA, Cochran DL, et al. Practitioner engagement in activities of the national dental practice-based research network (PBRN): 7-year results. J Am Board Fam Med. 2020 Sep-Oct;33(5):687-97.

23 Maxey HL, Norwood CW, Weaver DL. Primary care physician roles in health centers with oral health care units. J Am Board Fam Med. 2017;30(4):491-504.
24 Stoopler ET, Sollecito TP. Oral medicine in dermatology: an interprofessional partnership. Dermatol Clin. 2020;38(4):xi-xii.

25 Colleges AoAM. Report IX: contemporary issues in medicine: oral health education for medical and dental students. 2008.

26 Committee on an Oral Health Initiative BoHCS. Advancing oral health in America. Washington: Institute of Medicine; 2011.

27 American Dental Education Association Contemporary issues in medicine: oral health education for medical and dental students. Bull Dent Educ. 2011;44(10).

28 Stoopler ET, Shirlaw P, Arvind M, Lo Russo L, Bez C, De Rossi S, et al. An international survey of oral medicine practice: proceedings from the 5th World workshop in oral medicine. Oral Dis. 2011;17(Suppl 1): 99-104

29 Whitney EM, Stoopler E, Brennan MT, DeRossi SS, Treister NS. Competencies for the new postdoctoral oral medicine graduate in the United States. Oral Surg Oral Med Oral Pathol Oral Radiol. 2015;120(3):324-8.

30 Stoopler ETDe Rossi SS, Greenberg MS, Sollecito TP. Using scholarly productivity as an outcome assessment of a dental residency program. Spec Care Dentist. 2020;40(6):616-

31 Stoopler E. Oral medicine achieves specialty recognition by the American dental association. J Orofac Sci. 2020;12(1):1-2.

32 National Commission on Recognition of Dental Specialties and Certifying Boards [cited 2020 October 21]. Available from: https:// www.ada.org/en/ncrdscb/who-we-are/newsand-meetings.

33 Spielman AI. The future of oral medicine. Oral Dis. 2018;24(1-2):285-8.

34 Alrashdan MS, Alazzam M, Alkhader M, Phillips C. Career perspectives of senior dental students from different backgrounds at a single Middle Eastern institution. BMC Med Educ. 2018;18(1):283.

35 Khoury ZH, Sultan AS. The state of oral medicine and oral pathology in the Arab Middle East. Saudi Dent J. 2019.

36 Tyler MT, Miller CS, Lockhart PB, Patton LL. American academy of oral medicine: 75 years of bringing medicine and dentistry back together. Oral Surg Oral Med Oral Pathol Oral Radiol. 2020;129(2):91-4. 